\title{
THE EFFECT OF COMPOSITION MODIFICATION ON THE OPTICAL POLARIZATION INDEPENDENCE IN SEMICONDUCTOR STRAIN QUANTUM WELLS
}

\section{Wallace C.H. CHOY, Hao FENG, S.K. KAM and E. Herbert LI}

Department of Electrical and Electronic Engineering, University of Hong Kong, Pokfulam Road, Hong Kong. ehli@hkueee.hku.hk

\section{ABSTRACT}

Polarization independent quantum well (QW) materials operating under electroabsorption effect in optical switching and modulation devices are of intense interest recently. This is a theoretical analysis of the optical properties of strained $\mathrm{InGaAs} / \mathrm{InP} \mathrm{QWs}$. The method of composition modification based on interdiffusion will be introduced to merge the heavy- and light- hole states in order to achieve polarization insensitivity. Results presented here show that the diffused QWs with and without as-growth tensile strain can both serve in polarization independent electro-absorption requirements. With a suitable design in the interdiffesed $\mathrm{QW}$ materials, the optical polarization independence can operate from 1.465 to $1.540 \mu \mathrm{m}$ (tunability of $75 \mathrm{~nm}$ ) with a maximum absorption change of $2000 \mathrm{~cm}^{-1}$. In the case studied here, over $75 \%$ reduction in the required as-growth tensile strain is achieved as compared with the conventional rectangular QWs. This provides us with a simpler way to achieve high strain optical polarization independence through interdiffusion.

\section{INTRODUCTION}

In the realization of monolithical optoelectronic integration [1], large refractive change, large absorption change, optical switching and modulation employing quantum wells $(\mathrm{QW})$ are very attractive. However, these $\mathrm{QW}$ structures bear various drawbacks including a strong polarization dependence in optical fields [2]. Recently, polarization independence or insensitive electro-absorptive modulation has been widely investigated in different regular quantum well (QW) material systems [3]. This is the first theoretical study using interdiffusion technique to generate the tensile (in well) and compressive (in barrier) effects for the InGaAs/InP material system in hopes of achieving polarization independence. The main principle behind achieving polarization independence in QWs is to tailor an identical change in absorption coefficient for both TE and TM mode optical polarization light (parallel and perpendicular to the plane of QWs respectively) while the $\mathrm{QW}$ is under bias. The identical change in absorption of the two modes can be obtained by inducing tensile strain with the value of $0.43 \%$ [4] in wells of $\operatorname{InGaAs} / \mathrm{InP}$ QW. However, fabricating such a large initial strain QW remains a challenge. Interdiffused Quantum well (DFQW) technology is one of the methods which enables us to overcome the barrier in developing the strainer material structure, while at the same time achieving polarization independence in electro-absorptive modulation. Moreover, there are two possible ways to obtain polarization independent InGaAs/InP DFQWs in our studies. The first one is to start with a lattice matched QW system together with the strain effects which are mainly induced by interdiffusion. The second method is by generating a small initial tensile strain in the wells and by enhancing the strain through suitable interdiffusion.

In this paper, a DFQW structure which performs as a polarization independent electroabsorptive modulator will be discussed. This consists of four sections: the first discusses the chronological steps in assessing polarization independence, the second discusses the effect of 
initial $P$ concentration in the InGaAsP well, while the last two sections contain discussions of the DFQW properties with and without as-growth tensile strain in the wells. Finally a conclusion is drawn.

\section{MODEL}

The polarization dependent absorption coefficient of the DFQW is calculated for the $\Gamma$ region of the Brillouin zone. The major contribution to the change of absorption, $\Delta \alpha$, arises from transitions of the $1 \mathrm{~S}$ exciton and the lower energy bound states. (Details of the discussion are illustrated in reference 5). The interdiffusion of InGaAs/InP material system has been studied by using diffusion equations [6]. There are three possible ways of interdiffusions: (a) Group III (In, Ga atoms) diffusion only, (b) Group V (As, P) diffusion only, and (c) Group III and V diffusion. In order to induce tensile strain large enough for polarization independence, Group V diffusion is considered, since option (a) can only induce compression strain while option (c) can only investigate weak tensile or compressive strain. The group $\mathrm{V}$ interdiffusion processes can be modeled by a two-phase diffusion system with two diffusion coefficients in the substrate: and the well layer [7] and incorporating discontinuous concentrations across the QW boundary. The diffusion has been thoroughly discussed in [6]. An initial lattice match $\operatorname{In}_{x} G_{1-x} A s_{y} P_{1-y} / \operatorname{InP}$ system can be fabricated when the relation $y=2.2(1-x)$ holds [8]. The various interdiffusions are indicated by the diffusion time and diffusion temperature.

The lattice matched InGaAs/InP QW system is polarization sensitive because of the splitting of first state heavy hole (hh) and light hole (lh). In addition, the oscillator strength of state (e) in the conduction band and hh is different from that of e and $\mathrm{lh}[4,9]$. While Quantum Confined Stark Effect is applied to the material system, the red shift of the interband transition energy of e-hh and e-lh are different from each other since the heavy hole and light hole have different effective mass [10]. So TE and TM mode optical polarization depends on the transition of e-hh together with e-lh. These have different variations in electro-optical properties including electro-refraction and electro-absorption. In order to acquire the polarization insensitivity, the splitting of the first eigenstates of hh and lh have to be removed. In fact, a suitable tensile strain can fulfill this requirement. The dominating parameters to adjust tensile strain of the DFQW are the composition ratio of the elements of the material system, well width, diffusion temperature, diffusion time, broadening factor (both exciton and bound states), and applied field. The first parameter, composition ratio, will generate an initial tensile strain on the wells. The second, concentration ratio of indium in the wells, is adjusted to increase the initial tensile strain. The well width determines the initial splitting of $\mathrm{th}$ and hh. While the well width increases, the splitting decreases. The diffusion temperature and diffusion time are varied to further enlarge the tensile strain on the wells for the polarization independence. We will use a lower energy tail of the band edge (with respect to the exciton peak) to achieve polarization independent because the oscillation strengths of $\mathrm{lh}$ and hh are different at the exciton peak. Also, the broadening factor of the material system highly affects the independent properties. The broadening factor is a function of: the energy states of $\mathrm{lh}$ and $\mathrm{hh}$, the field applied to the material, the extent of interdiffusion, the strain induced, and the well width. Since there is no well agreement between measured data in the broadening factor from experiments [13-16], we have determined this factor in according to the following conditions. In the increment of field, interdiffusion, temperature, strain and well width, the broadening factor increases. Moreover, the broadening factor of $\mathrm{lh}$ is larger than that of hh. It should be noted that the applied field is maximized under the requirement to maintain the polarization independence. Therefore the operation wavelength can 
operate far from the high residence loss wavelength range (the range of exciton peak under no bias).

While the $\operatorname{InGaAs} / \mathrm{InP}$ is under Group $\mathrm{V}$ interdiffusion, the two phase diffusions will change the compositional profiles (As and $P$ ). This variation has strong tensile effect in the system. Any deviation in concentration from the as-grown lattice will affect the bandgap energy. The amount of lattice mismatching governed by the concentration profile contributes a perturbed strain energy toward the Hamiltonian, in the Schrödinger equation of the material system, which modulates the confined energy levels. The variation of concentration profile in turn affect on the value of the carrier effective mass, the bulk bandgap and the confinement profile. These effects adjust the states of $\mathrm{lh}$ and $\mathrm{hh}$. In polarization independence, the shear strain acted on the $\mathrm{lh}$ and hh states will force them together when the relation $2 \delta \mathrm{E}_{\mathrm{s}}=\delta \mathrm{E}_{\mathrm{lh}}-\delta \mathrm{E}_{\mathrm{hh}}$ holds, where $\delta \mathrm{E}_{\mathrm{s}}$ is the shear deformation potential, and $\delta \mathrm{E}_{\mathrm{lh}}$ and $\delta \mathrm{E}_{\mathrm{hh}}$ are the light- and heavy-hole energy levels respectively. This is one of the crucial criterion to the TE and TM optical polarization sensitivity in the QW structures.

\section{RESULTS AND DISCUSSION}

After a theoretical study of the factors toward polarization insensitivity, we draw out here the chronological steps for achieving polarization independence. The first step is to remove the splitting effect between $\mathrm{lh}$ and hh. This means that the initial tensile strain, diffusion temperature and diffusion time should be adjusted. The second step is to set the field as high as possible, in order to develop a low residence loss electro-absorptive modulation. The third step is to modify the well width, in order to satisfy the high field requirement, while maintaining a minimum splitting effect of $\mathrm{lh}$ and hh. The smaller the as-grown (initial) strain the more extensive the interdiffusion will be required and therefore larger diffusion times and temperatures are expected. The final step is to determine the broadening factors so that the change of absorption coefficient of TE and TM mode is the same.

The initial concentration (1-y) of $P$ in the well $\left(\operatorname{In}_{x} G_{1-x} A s_{y} P_{1-y}\right)$ will increase the splitting between $\mathrm{lh}$ and $\mathrm{hh}$. This means that larger tensile strains are required to pull the $\mathrm{lh}$ and hh states together. Thus, more extensive interdiffusion is required (implies higher diffusion temperature or larger diffusion time). The interdiffusion can be reduced by introducing initial tensile strain to the QW structure before any interdiffusion. The effect of $P$ in the well will only enhance the difficulty (high tensile strain for merging $\mathrm{lh}$ and $\mathrm{hh}$ ) in obtaining polarization independence. Therefore the InGaAs/InP QW material system with no $\mathrm{P}$ in the wells prior to interdiffusion is selected.

An initial tensile strain can be generated in the as-grown QW by reducing the concentration of indium $(<0.53)$ in the well. Two different concentration ratio of $x=0.511$ and $\mathrm{x}=0.506$ are investigated. Fig. 1 , shows the difference between $\mathrm{lh}$ and hh transition energies gradually reduces with increasing diffusion time because larger tensile strain is obtained under large interdiffusion. Fig 1 (a) and 1 (b) show that the cas $s$ of $x=0.511$ and $x=0.506$ merging of $\mathrm{lh}$ and hh can be achieved, with diffusion temperature or $800^{\circ} \mathrm{C}$ and $750^{\circ} \mathrm{C}$, respectively, at corresponding diffusion times of 2.35 hours and 2.83 bours. The applied field for both of these two cases is $130 \mathrm{kV} / \mathrm{cm}$. In fact, the polarization independent electro-absorptive modulator will operate under applied fields of 0 and $130 \mathrm{kV} / \mathrm{cm}$. The $\Delta$, of TE and TM modes are shown in Fig 2(a) and (b). For polarization independence, it shoulc operate in situation that $\Delta \alpha_{\mathrm{TE}}=\Delta \alpha_{\mathrm{TM}}$ while $\alpha$ is chosen as small as possible. This can be best achieved in the case of $x=0.511$ between $\lambda=1.47$ and $1.52 \mu \mathrm{m}$ and, in the case of $\mathrm{x}=0.506$, between $\lambda=1.465$ and $1.54 \mu \mathrm{m}$. The 


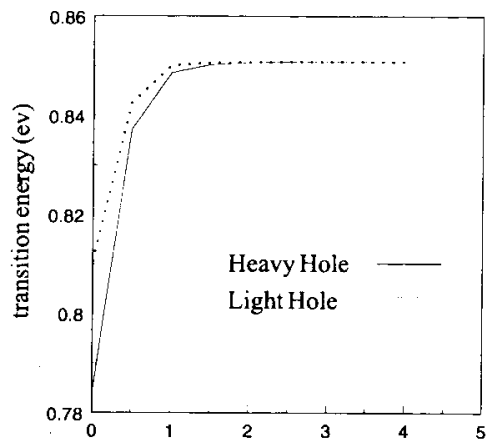

Fig 1(a)

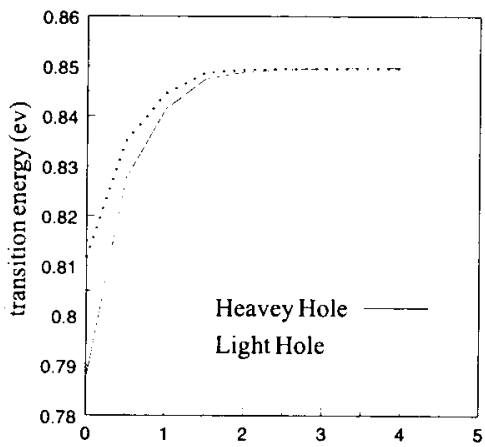

Fig 1(b)

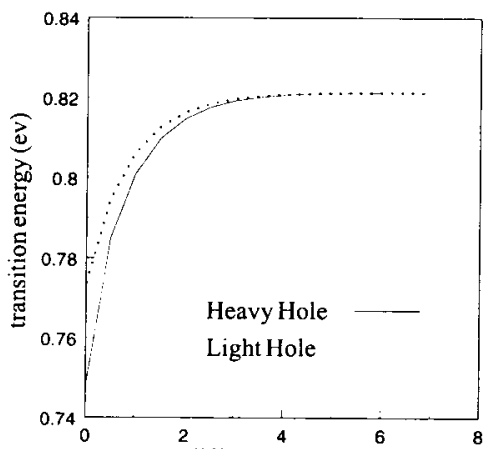

Fig 1(c) diffusion time (hr)

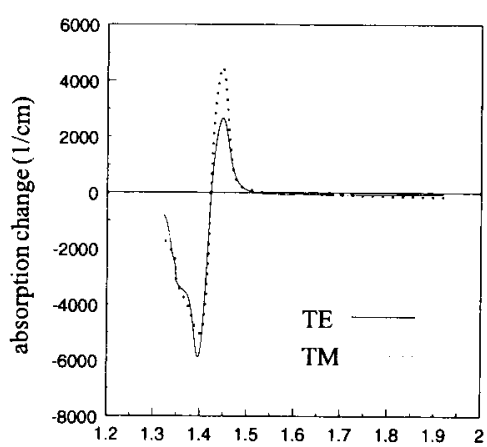

Fig 2(a)

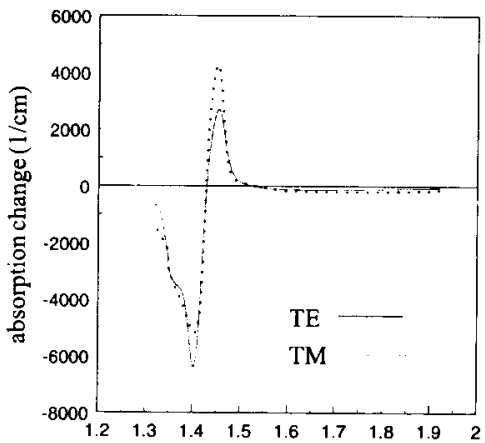

Fig 2(b)

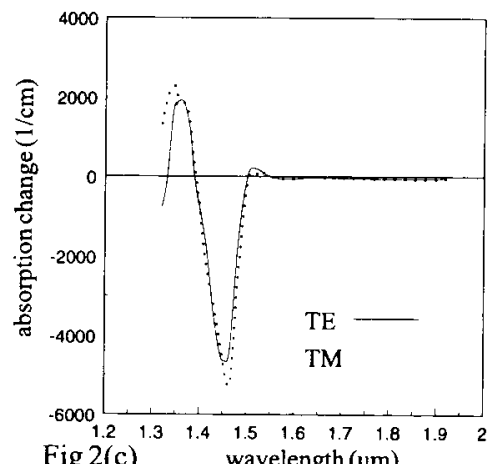

Fig. 1 The change of transition energy of $\mathrm{HH}$ (solid line) and $\mathrm{LH}$ with diffusion time (a) $0.145 \%$ as-grown tensile strain induced in the $\operatorname{In}(0.511) \mathrm{Ga}(0.498) \mathrm{As} / \mathrm{InP}$ DFQW with well width $(\mathrm{Lz})=10 \mathrm{~nm}$, the transition energy of LH and $\mathrm{HH}$ matched after 2.35 hours interdiffusion . (b) $0.18 \%$ as-grown tensile strain induced in the $\operatorname{In}(0.506) \mathrm{Ga}(0.494) \mathrm{As} / \mathrm{InP}$ DFQW with $\mathrm{Lz}=10 \mathrm{~nm}$. The transition energy of LH and $\mathrm{HH}$ matched after 5.01 hours interdiffusion. (c) no as-grown strain induced in the $\operatorname{In}(0.53) \mathrm{Ga}(0.47) \mathrm{As} / \mathrm{InP} \mathrm{DFQW}$ with $\mathrm{Lz}=$ $15 \mathrm{~nm}$, the transition energy of $\mathrm{LH}$ and $\mathrm{HH}$ matched after 5.01 hours interdiffusion.

Fig. 2 The change of absorptin coefficient of TE (solid line) and TM (dashed-line) modes for the three respective cases in Fig. 1. 
maximum $\Delta \alpha$ of these two cases is about $2000 \mathrm{~cm}^{-1}$. Although this situation can also be found in the other wavelength ranges, they cannot be implemented due to an unexpectedly large residual loss. The exciton broadening factor of hh $(\mathrm{lh})$ under zero and $130 \mathrm{kV} / \mathrm{cm}$ field in the case of $x=$ 0.511 are taken as $9 \mathrm{meV}(39 \mathrm{meV})$ and $10 \mathrm{meV}(42 \mathrm{meV})$ respectively. When $\mathrm{x}=0.506$, the corresponding broadening factors of hh $(\mathrm{lh})$ are $9 \mathrm{meV}(40 \mathrm{meV})$ under zero field and $10 \mathrm{meV}$ $(43 \mathrm{meV})$ under $130 \mathrm{kV} / \mathrm{cm}$. The broadening factors are similar in the two cases because they have the same well width $(100 \AA)$ and operating under the same bias. The case of $x=0.511$ has a smaller strain than the case of $x=0.506$, however, it can be enhanced by a relatively extensive interdiffusion through a higher annealing temperature to create two order of magnitude larger in the diffusion coefficient. As shown in Fig 1(c), the lattice matched material system of $x=0.53$ with zero initial strain can create enough tensile strain to perform polarization insensitivity after interdiffusion. The conditions for this interdiffusion with $150 \AA$ well width is to anneal at a temperature of $800^{\circ} \mathrm{C}$. The exciton broadening factor of hh (lh) with and without field are taken to be $11 \mathrm{meV}(19 \mathrm{meV})$ and $12 \mathrm{meV}(22 \mathrm{meV})$, respectively. The maximum $\Delta \alpha$ value falls within the selected operation wavelength range from 1.526 to $1.56 \mu \mathrm{m}$ is about $250 \mathrm{~cm}^{-1}$, which is a degradation comparing to the previous two cases. However, correspondingly, the maximum applied field $(70 \mathrm{kV} / \mathrm{cm})$ and tensile strain $(0.252 \%)$ are improved over the previous two cases with $0.325 \%$ strain. This reduction can be explained by the increase of well width from $100 \AA$ (in the case of $x=0.511$ ) to $150 \AA$ (in the present case) which contributes to a reduced splitting effect between $\mathrm{lh}$ and hh. It should be noted that the annealing temperature of the third case is the same as that of the case of $x=0.511$

Variation of Strain (in\%) with wellwidth

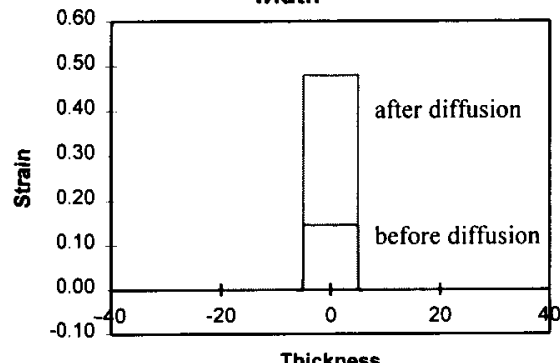

Fig 3(a)

\section{Variation of Strain (in \%) with well- width}

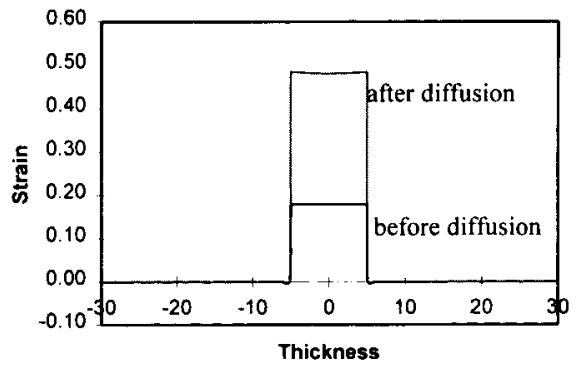

Fig 3(b)

Figure 3. The strain induced in wells and barriers of the material system before and after the interdiffusion mentioned in the first two cases in Figure 1.

The strain, as shown in Fig 3, in the DFQW cases of $x=0.511$ and $x=0.506$ after interdiffusion is $0.481 \%$ and $0.485 \%$, res petively, while their respective as-grown strain are small $(0.145 \%$ and $0.18 \%)$ in comparison to the rectangular $100 \AA / 100 \AA \mathrm{QW}$ (without diffusion at all), which requires $0.43 \%$ as-grown strain for the polarization independence[4]. In the case of $\mathrm{x}=0.511$, there is a reduction of more than $66 \%$ in tensile strain by using the DFQW material in achieving polarization independence. As a consquence, the difficulty in fabrication is highly reduced. Moreover, from Fig 3(a) and (b), the compressive strain generated outside is small enough (with order $10^{-7}$ and $10^{-4}$ in cases of $x=0.511$ and $x=0.506$ respectively) to be ignored. 
Therefore, the group V two-phase interdiffusion only generates tensile strain in the wells but without any effect on the barrier.

\section{CONCLUSION}

We have investigated the polarization independence of the electro-absorptive property in InGaAs/InP DFQW. It is shown that the required as-grown tensile strain can be reduced by $66 \%$ if an optimized DFQW structure is employed. An operation wavelength ranging from 1.465 to $1.54 \mu \mathrm{m}$ (tunability of $75 \mathrm{~nm}$ ) is obtained and a simple technology (low strain growth plus postgrowth annealing) is realized to provide polarization independence. This independence characteristic can also be obtained by employing interdiffusion in the as-grown unstrained ( $\mathrm{x}=$ $0.53) \mathrm{QW}$ material. In order to reduce the tensile strain required $(0.252 \%)$ to merge the $\mathrm{lh}$ and hh states, the well width is enlarged to $15 \mathrm{~nm}$. However, this enlargement will introduce drawbacks such as a narrower operation wavelength range and lower $\Delta \alpha$ values. It is therefore concluded that DFQW with small as-growth tensile strain will be more useful to produce polarization insensitivity in electro-absorption modulation applications.

\section{ACKNOWLEDGMENTS}

The authors would like to thank the University of Hong Kong - Committee on Research and Conference Grants (CRCG) and the University Grants Council (HK) - RGC Earmarked Research Grant for financial support. W.C.H. Choy would like to express his gratitude for the financial support of the Sir Edward Youde Memorial Fellowship.

\section{REFERENCES}

1. H. Yamamoto, M. Asada and Y. Snematsu, J. Lightwave Technol. 6, 1831 (1988).

2. K. Wakita, Y. Kawamura, M. Nabeto, and H. Asai, IEEE J. Quantum Electron. 23, 2210 (1987).

3. Y. C. Chau and K. Tada, IEEE Photon. Technol. Lett. 5, 1380 (1993).

4. T. Aizawa, K. G. Ravikumar, S. Suzaki, T. Watanabe, and R. Yamanchi, IEEE J. Quantum Electron. 30, 585 (1994).

5. E. H. Li and B. L. Weiss, IEEE Photon Technol. Lett. 4, 445 (1993).

6. K. Mukai, M. Sugawara, and S. Yamazaki, Phys. Rev. B 50, 2273 (1994).

7. T. Fujii, M. Sugawara, S. Yazaki and K. Nakajima, J. Crys. G. 105, 348 (1990).

8. J. Micallef, E. H. Li, and B. L. Weiss, J. Appl. Phys., 73, 7524 (1993).

9. T. C. Chong, H. W. Wan, and S. J. Chua, Electron. Lett. 26, 1060 (1990).

10. T. Ishikawa, S. Nishimura and K. Tada, Japan. J. Appl. Phys. 29, 1466 (1990).

11. J. C. Zucker, K. L. Jones, T. H. Chiu, B. Tell and K. B. Goebeler, IEEE J. Lightwave Technol. 10, 1926 (1992).

12. T. Yamaaguchi, T. Morimoto, Y. Nakano, and K. Tada, Technical Digest, Topcial Meeting on Integrated Photonics Research (IPR' 94), FFG, 263 (1994).

13. K. Tai, K. J. Hegarty, and W. T. Tsang, Appl. Phys. Lett. 51, 152 (1987).

14. Y. G. Kawaguchi and H. Asahi, Appl. Phys. Lett. 50, 1243 (1987).

15. C. P. Kuo, K. L. Fry, and G. B. Stringfellow, Appl. Phys. Lett. 47, 885 (1985).

16. J. H. Marsh, J. S. Roberts, and P. A. Calxton, Appl. Phys. Lett. 46, 1161 (1985). 